ORIGINAL ARTICLE

\title{
Idiopathic perianal pruritus: washing compared with topical corticosteroids
}

\section{O Öztaș, P Öztaș, M Önder}

See end of article for authors' affiliations

......................

Correspondence to: Dr Murat Orhan Öztas, Gazi Universitesi Tip Fakültesi, Dermatoloii Anabilim Dalı, Besevler, Ankara, Turkey; moztas@gazi.edu.tr

Submitted 25 July 2003 Accepted

8 September 2003
Postgrad Med J 2004;80:295-297. doi: 10.1136/pgmi.2003.013045

Background: Idiopathic perianal pruritus is a poorly managed condition. Topical corticosteroids are effective in idiopathic perianal pruritus, but they may cause some side effects. In this study, the effectiveness of topical steroids were compared with perianal cleansers in the treatment of idiopathic perianal pruritus. Patients and methods: Seventy two patients with the complaint of perianal pruritus were evaluated; 60 of the 72 patients were found to be idiopathic. Twenty eight patients were treated with topical steroids and 32 patients were treated only with a liquid cleanser.

Results: At the end of this two week period, cleansers were found to be as effective as topical corticosteroids.

Conclusion: This study shows that perianal cleansers can be used as a safe first step treatment in idiopathic perianal pruritus.
$\mathrm{P}$ erianal pruritus is a common symptom, defined as an unpleasant cutaneous sensation that induces scratching of the skin around the anal orifice. ${ }^{1}$ Itchiness of the perianal skin can be a dermatological, proctological, or psychological disorder. ${ }^{2}$ Persistent perianal pruritus is a generally poorly managed, common, and socially embarrassing condition. ${ }^{3}$ There are many different causes of perianal pruritus such as haemorrhoids, anal fistula and fissures, inflammatory bowel disease, dermatoses (atopic dermatitis, psoriasis, lichen planus, Bowen's disease, extramammary Paget's disease, allergic contact dermatitis), local infections (candidiasis, dermatophyte infections, parasites, condyloma lata), benign and malignant anal tumours, generalised pruritus, foods (spices, citrus fruits, coffee, tea, chocolate, tomatoes), and psychogenic causes (table 1). The most difficult patients are the ones with extensive pruritus without physical signs..$^{3-5}$ When no demonstrable cause is found, it is often described as idiopathic perianal pruritus. ${ }^{3}$ There are many treatment modalities for idiopathic perianal pruritus, including topical corticosteroids, topical non-steroid anaesthetics, and systemic antihistamines. ${ }^{67}$ Topical corticosteroids are known to be effective for non-fungal types of perianal pruritus. ${ }^{6}$ In this study, we tried to compare the effectiveness of topical steroids and perianal cleansing in the treatment of idiopathic perianal pruritus.

\section{PATIENTS AND METHODS}

Seventy two patients who presented to Gazi University Hospital Department of Dermatology with the complaint of perianal pruritus over a 24 month period were evaluated. All of the patients were first examined dermatologically. From perianal scrapings, native preparations were performed for fungal investigation. Perianal swabs for bacterial cultures were also performed. Complete blood counts, fasting glucose levels, liver and kidney function (alanine aminotransferase, aspartate aminotransferase, $\gamma$-glutamyltransferase, lactate dehydrogenase, blood urea nitrogen, creatinine), and total cholesterol and triglyceride levels were recorded. Investigation of faeces for parasites was also performed. In 11 patients, allergic contact dermatitis was suspected clinically and patch tests with both European Standard Series and personal items were performed. In 12 patients the causes of perianal pruritus were detected, and these patients with a defined cause were not included in our study. Sixty patients were classified as having "idiopathic perianal pruritus patients". Twenty eight patients (group I) were treated with topical steroids (Advantan cream) twice daily for two weeks, and 32 patients (group II) were treated only with a liquid cleanser (Protex liquid cleanser) twice daily for two weeks. At the end of the two week period, the patients were re-examined. In both examinations (before and after treatment), patients were requested to score their pruritus in the range of 0-10 (0: no pruritus, 10: the most severe pruritus).

The $\chi^{2}$ test was used for statistical significance. Statistical analysis was performed using software SPSS 11.0 (Statistical Package for Social Science 11.0); a p value less than 0.05 was considered significant.

\section{RESULTS}

In 12 patients, the cause of perianal pruritus was detected (haemorrhoids, $\mathrm{n}=4$; anal fissure, $\mathrm{n}=1$; Enterobius vermicularis infestation, $\mathrm{n}=1$; dermatophyte infection, $\mathrm{n}=1$; condyloma accuminata, $\mathrm{n}=1$; perianal molluscum contagiosum, $\mathrm{n}=1$; diabetes mellitus, $\mathrm{n}=1$; and psychogenic factors, $\mathrm{n}=2$ ) and these patients were excluded from the study (table 2). In 11 patients, allergic contact dermatitis was suspected clinically and patch tests with both European Standard Series and personal items were performed. No allergic or irritant reaction was seen in these patients.

The mean (SD) age of the idiopathic perianal pruritus patients was 34.78 (16.68); 35 patients were male (58.3\%) and 25 were female $(41.7 \%)$. Twenty eight patients (mean (SD) age 35.43 (16.32), 16 male, 12 female) were treated with topical steroid (Advantan cream; Schering) twice daily and treatment was effective in $26(92.3 \%)$. Thirty two patients (mean (SD) age 34.20 (15.35), 19 male, 13 female) were treated with a liquid cleanser (Protex liquid cleanse; ColgatePalmolive) and treatment was effective in 29 (90.6\%). The difference between the effectiveness of these two treatment modalities was not statistically significant $\left(\chi^{2} \mathrm{p}>0.05\right)$.

\section{DISCUSSION}

Perianal pruritus is an extremely common symptom and is associated with a wide range of mechanical, dermatological, infectious, systemic, and other conditions. ${ }^{8}$ 
Table 1 Aetiological factors in perianal pruritus

Idiopathic perianal pruritus

Secondary perianal pruritus

- Dermatological disorders (atopic dermatitis, allergic contact dermatitis, psoriasis, lichen sclerosus, extramammary Paget's disease, squamous cell carcinoma).

- Infections

- Bacterial (Staphylococcus aureus, group A and B streptococcus).

- Intertrigo.

- Fungal (candidiasis, dermatophytosis).

- Parasitic (Enterobius vermicularis, scabies).

- Sexually transmitted diseases (herpes simplex virus infection, syphilis, condyloma accuminata)

- Local irritants (faecal contamination, sweating).

- Systemic disorders (diabetes mellitus, lymphoma, psychogenic factors).

Adapted from Johnson.

Inspection, palpation, and anoscopic examination should be performed to find out the aetiology. In this study, a total of 72 patients suffering from perianal pruritus were examined. The aetiology of perianal pruritus was found in only $12(20 \%)$ of our patients.

Haemorrhoids and anal fissures are important causes of perianal pruritus. Daniel et al diagnosed haemorrhoids in $20 \%$ and anal fissures in $12 \%$ of patients with perianal pruritus. ${ }^{8} 9$ During our study, we diagnosed haemorrhoids in four patients and anal fissure in one patient, which were excluded from statistically analysed "idiopathic perianal pruritus" group.

There are many other organic and metabolic factors of perianal pruritus such as intestinal parasites, perianal fungal, bacterial and viral infections, and diabetes mellitus. It is important to treat the underlying aetiological factors, if detected In our study, "Enterobius, vermicularis infestation" was detected in a 5.5 year old girl. In one patient, perianal molluscum contagiosum was present. Perianal condyloma accuminata was diagnosed in another patient. Diabetes mellitus was diagnosed in one patient and in another dermatophyte infection was found with direct microscopic investigation.

Psychological problems are well known causes of perianal pruritus. Laurent et al showed that the mean hypomania and depression scale scores were greater and smaller respectively in the idiopathic perianal pruritus patients. ${ }^{10}$ In two of our patients, depression was diagnosed after psychiatric consultation.

There are many other factors known to initiate or aggravate perianal pruritus. It is very important to remember that some foods, such as spices, citrus fruits, coffee, tea, chocolate, and tomatoes can cause perianal pruritus. ${ }^{45}$ Such

\begin{tabular}{ll}
$\begin{array}{l}\text { Table } 2 \text { Detected causes of perianal pruritus in } \\
\text { our study }\end{array}$ \\
\hline Aetiology & No of cases \\
\hline Haemorrhoids & 4 \\
Anal fissure & 1 \\
Fungal infection & 1 \\
Condyloma accuminata & 1 \\
Parasitic infestations & 1 \\
Molluscum contagiosum & 1 \\
Diabetes mellitus & 1 \\
Psychogenic factors & 2 \\
Idiopathic & 60 \\
Total & 72 \\
\hline
\end{tabular}

Components of topical steroid and liquid

cleanser

- Advantan cream: methylprednisolone aceponate $0.1 \%$.

- Protex liquid cleanser: sodium lauryl ether sulphate; DMDM hidantoine; glycerin; cocodiethanolamide; triclosan; aloe vera extract; cocoamidoprpyl betaine; citric acid; EDTA; $\mathrm{NaCl}$.

foods should be eliminated from the diets of patients as part of the treatment. ${ }^{511} 12$

Faecal contamination is also very important in perianal pruritus. ${ }^{13}{ }^{14}$ Faeces contain endopeptidases of bacterial origin, in addition to potential allergens and bacteria. Although Silverman et al could not show a microbiological basis for perianal pruritus, ${ }^{15}$ these enzymes are capable of both itching and inflammation..$^{13}$ At that point, we decided to investigate the role of decreasing faecal contaminants with cleansers and find out the effectiveness of washing in the treatment of perianal pruritus.

If a specific cause of perianal pruritus is identified, appropriate therapy such as antifungals, antibiotics, and antihelmintics should be given. ${ }^{6}$ There are many treatment modalities for idiopathic perianal pruritus, including topical corticosteroids, topical non-steroid anesthetics, and systemic antihistamines. ${ }^{67}$ Topical corticosteroids are widely used and they are one of the most effective treatment regimens for perianal idiopathic pruritus. ${ }^{6}$

In this study, we tried to compare the effectiveness of perianal cleansing and topical corticosteroids in the treatment of idiopathic perianal pruritus. Symptoms of 26 (92.9\%) patients in group I, and $29(90.6 \%)$ patients in group II improved. No side effects in either group were detected at the end of the treatment period. Although topical corticosteroids are one of the most effective treatment regimens for perianal idiopathic pruritus, the skin of anogenital area is particularly vulnerable to atrophy after the use of such medications. ${ }^{6}{ }^{16}$ Although we did not detect such complications, topical corticosteroids, especially if used for a long period, can cause atrophy, bacterial and fungal infections, allergic contact dermatitis, telengectasia, purpura, and/or scar formation. ${ }^{17}$

In conclusion, this study shows that perianal cleansing is as effective as topical corticosteroids in the treatment of idiopathic perianal pruritus, and mild cleansers can be used as a safe first step treatment for controlling perianal itching.

\section{Authors' affiliations}

M O Öztas, M Önder, Department of Dermatology, Gazi University Faculty of Medicine, Ankara, Turkey

P Öztaș, Dermatology Clinic, Ankara Numune Education Hospital, Ankara, Turkey

\section{REFERENCES}

1 Sullivan ES, Garnjobst WM. Symposium on colon and anorectal surgery. Pruritus ani: a practical approach. Surg Clin North Am 1978;58:505-12.

2 Alexander-Williams J. Pruritus ani. BMJ 1983;287:159-60.

3 Dasan S, Neill SM, Donaldson DR, et al. Treatment of persistent pruritus ani in a combined colorectal and dermatological clinic. Br J Surg 1999:86:1337-40

4 Braun Falco O, Plewig G, Wolff HH, et al. Dermatologic proctology. In: Braun Falco O, Plewig G, Wolff HH, et al. Dermatology. 2nd Ed. Berlin: Springer, 2000:1693-700.

5 Aucoin EJ. Pruritus ani. Postgrad Med 1987;82:76-80.

6 Odom RB, James WD, Berger TB. Pruritus and neurocutaneous dermatoses. In: Odom RB, James WD, Berger TB, eds. Andrews' diseases of the skin. 9th Ed. Philadelphia: Saunders, 2000:49-68.

7 Johnson RA. Diseases and disorders of the anogenitalia of males. In: Freedberg IM, Eisen AZ, Wolff K, et al, eds. Fitzpatrick's 
dermatology in general medicine. 5th Ed. New York: Mc Graw-Hill, 1999:1348-76

8 Pfenninger JL, Zainea GG. Common anorectal conditions: part I. Symptoms and complaints. Am Fam Physician 2001;63:2391-8.

9 Daniel GL, Longo WE, Vernava AM 3rd. Pruritus ani. Causes and concerns. Dis Colon Rectum 1994;37:670-4.

10 Laurent A, Boucharlat J, Bosson JL, et al. Psychological assessment of patients with idiopathic pruritus ani. Psychother Psychosom 1997;66:163-6.

11 Giordano M, Rebesco B, Torelli I, et al. Pruritus ani. Minerva Chir 1999:54:885-91.

12 Smith LE, Henrichs D, McCullah RD. Prospective studies on the etiology and treatment of pruritus ani. Dis Colon Rectum 1982;25:358-63.
13 Ive FA. The umbilical, perianal and genital regions. In: Champion $\mathrm{RH}$, Burton JL, Burns DA, et al, eds. Textbook of dermatology. 6th Ed. Oxford: Blackwell-Science, 1998:3163-238.

14 Kocsard E. Pruritus ani. A symptom of fecal contamination. Cutis $1981 ; 27: 518$.

15 Silverman SH, Youngs DJ, Allan A, et al. The fecal microflora in pruritus ani. Dis Colon Rectum 1989;32:466-8.

16 Lyell A. Psychogenic and neurogenic skin disorders. In: Orkin M, Maibach HI, Dahl MV, eds. Dermatology. 1 st Ed. Connecticut: Appleton \& Lange, 1991:70-7.

17 Habif TP. Topical therapy and topical corticosteroids. Clinical dermatology. 3rd Ed. Missouri: Mosby, 1996:25-44.

\section{IMAGES IN MEDICINE}

\section{Pseudoxanthoma elasticum}

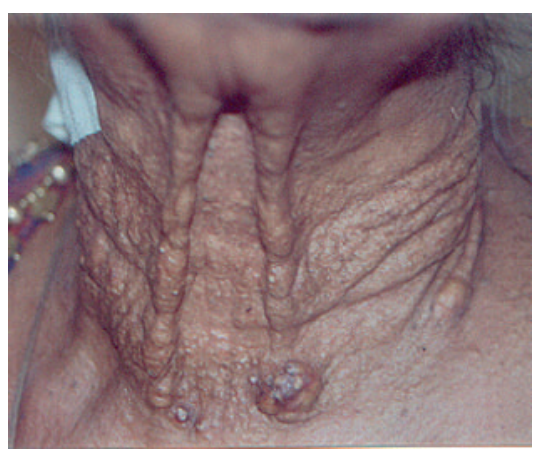

Figure 1 Loose skinfolds with cobblestone appearance of the skin of neck (published with patient's permission).
A 50 year old woman with progressive dimness of vision was referred by ophthalmologists for evaluation of her skin lesions. She had asymptomatic, yellowish, papular lesions on the sides and front of her neck, axillae, and periumbilical region. The lesions had been present since puberty and non-progressive for the past several years. She was hypertensive on irregular treatment. She had two uneventful pregnancies and the children were normal. Her mother and elder sister had similar skin lesions of long duration.

On examination, her peripheral pulses were normal. Supine blood pressure was 150/100 mm $\mathrm{Hg}$. The skin in the involved areas was lax and redundant with yellowish, waxy, papular lesions giving rise to a cobblestone appearance (fig 1). The inner aspect of her lips and buccal mucosa showed a yellowish pebbled surface. Ophthalmoscopy revealed bilateral choroiditis. With the above features, a clinical diagnosis of pseudoxanthoma elasticum was made. A skin biopsy was taken from a papular lesion on the neck. Elastic tissue stain (Verhoeff van-Gieson) of the histopathological section showed irregular clumps of elastic fibres in the mid-dermis along with areas of calcification. The features were consistent with pseudoxanthoma elasticum. The patient was sent for thorough cardiovascular evaluation.

Pseudoxanthoma elasticum is a rare inherited disorder of connective tissue affecting principally the dermis of skin, media, and intima of blood vessels and Bruch's membrane of the eye. The incidence of the disease is about one in 160000 . Two autosomal dominant and three autosomal recessive forms have been described with variable expression of the clinical features. In about $90 \%$ of the cases, the inheritance is autosomal recessive. The genetic defect of the disorder is located on chromosome 16p13.1. Histopathologically there is widespread elastorrhexis along with dystrophic calcification of the damaged elastic fibres.

Skin shows characteristic small, yellowish papules, present classically along the sides of neck, giving rise to "Moroccan leather" or "plucked chicken skin" appearance. Other sites of predilection are the axillae, periumbilical area, groin, perineum, and thigh. Similar lesions may occur in soft palate, inner aspect of the lips, and vagina. In older individuals, the involved skin is lax and hangs in large folds. Absence of skin lesions does not exclude pseudoxanthoma elasticum.

The commonest ocular change is the presence of angioid streaks and they are usually asymptomatic and occur in almost all patients with pseudoxanthoma elasticum. Progressive visual dimness, retinal haemorrhage, and occasionally total blindness may occur. Other associated ocular anomalies are drusen, leopard spotting, or a "string of pearls" appearance of the macula. Unaffected family members may have abnormal visibility of the choroidal vessels.

Generalised involvement of all large and medium sized arteries occurs. Manifestations of cardiovascular diseases may start in childhood as decreased peripheral pulses, intermittent claudication, hypertension, angina at younger age, myocardial infarction, and sudden death. Risk of cardiovascular diseases may be correlated with presence of angioid streaks.

Associations with Marfan's syndrome, scleroderma, and perforating elastosis have been reported. Hypercalcaemia, hyperphosphataemia, and abnormal metabolism of vitamin D are also associated biochemical abnormalities.

Diagnosis depends on clinical and histopathological features. Management is mostly preventive, reducing cardiac risk factors, taking care of ophthalmic and cardiovascular complications, screening of first degree relatives by ophthalmoscopy, and genetic counselling.

A C Inamadar, A Palit Department of Dermatology, Venereology and Leprology, BLDEA's SBMP Medical College, Hospital and Research Centre, Bijapur 586103, Karnataka, India; Aparuna1@rediffmail.com 